\title{
A Model of Contractile Tubules Showing How They Contract in the Heliozoan Echinosphaerium
}

\author{
Tatsuomi Matsuoka ${ }^{1}$, Yoshinobu Shigenaka ${ }^{2}$ and Yutaka Naitoh ${ }^{3}$ \\ ${ }^{1}$ Zoological Institute, Faculty of Science, ${ }^{2}$ Department of Information and \\ Behavioral Science, Faculty of Integrated Arts and Sciences, Hiroshima \\ University, Hiroshima 730, and ${ }^{3}$ Institute of Biological Science, University of \\ Tsukuba, Ibaraki 305, Japan
}

\begin{abstract}
In the large heliozoan Echinosphaerium, contractile tubules (formerly called X-bodies) are located between the axopodial membrane and the axonemal microtubules. When axopodial contraction occurs, the tubules have been thought to be transformed from a tubular to a granular form, as seen in ultra-thin sections. Our detailed morphological observations of the contractile tubules, however, have revealed that this so-called granulation of the contractile tubules is mediated by self-twisting and supercoiling during contraction.

We also examined the localization of calcium during axopodial contraction using a potassium pyroantimonate assay. $\mathrm{Ca}-\mathrm{Sb}$ deposits were detected on contractile tubules only during the twisting and coiling of this organelle. Our results indicate that axopodial contraction is enforced by the twisting and coiling of contractile tubules, which action probably is mediated by $\mathrm{Ca}^{2+}$ ions.
\end{abstract}

A heliozoan cell feeds on various kinds of protozoans and small-sized metazoans by conveying its prey, which become attached to the axopodial membrane, toward the cell body then engulfing it with a projection of the plasma membrane which makes a food cup. This ingestion of food organisms is performed by the rapid or slow contraction of axopodia and/or by axopodial flow (11). During axopodial contraction, moreover, axonemal microtubules are replaced by $\mathrm{C}$-shaped tubules $(9,11)$.

Rapid axopodial contraction has been reported to take place at a speed of $100 \mu \mathrm{m} / \mathrm{sec}$ or more as soon as a flagellate contacts with the distal portion of axopodium in Actinophrys sol (3). In Echinosphaerium akamae, however, contraction is induced even more rapidly, at a speed of about $800 \mu \mathrm{m} / \mathrm{sec}$, when activated charcoal particles become attached to the axopodium near its tip (in preparation). This rapid contraction of the axopodium probably is not be due only to the degradation of axonemal microtubules because they are cytoskeletal not contractile elements. Therefore, some type of contractile element must function in rapid axopodial contraction.

Suzaki et al. (11) suggested that the X-body (8) located inside the axopodium may be the contractile element which has a principal role in axopodial contraction and flow because it undergoes morphological change from the tubular to the granular

Reprint requests and all correspondence should be sent to Professor Y. Shigenaka, Faculty of Integrated Arts and Sciences, Hiroshima University, Hiroshima 730, Japan 
form when axopodia are stimulated externally by food organisms.

The detailed morphology of the contractile elements that operate in axopodial contraction has not been examined. Therefore, we investigated structural changes in contractile elements during axopodial contraction, and examined the change in $\mathrm{Ca}^{2+}$ localization within the axopodia. As a result, we propose a new term, "contractile tubules", instead of X-bodies the tentative name given by Shigenaka and Kaneda (8).

\section{MATERIALS AND METHODS}

The large heliozoan Echinosphaerium akamae (Strain HM) was cultured at $20 \pm 1^{\circ} \mathrm{C}$ in $0.01 \%$ Knop solution containing 1 or 2 wheat grains per $15 \mathrm{ml}$ of culture medium together with various food organisms that included Chilomonas paramecium and Tetrahymena thermophila. Prior to every experiment, the cultured heliozoans were washed in fresh medium and left for 1-2 $\mathrm{h}$ to let them adapt. To induce axopodial contraction, one of the food organisms, Chilomonas paramecium, was added at a high density $(60,000 \mathrm{cells} / \mathrm{ml})$ to the heliozoan-containing medium. After 1-2 sec, the heliozoans were fixed for $5 \mathrm{~min}$ with glutaraldehyde (GA) fixative containing $48 \mathrm{mM}$ phosphate buffer ( $\mathrm{pH} 7.0$ ), $0.02 \mathrm{mM} \mathrm{MgSO}_{4}$, $2 \mathrm{mM}$ sucrose, $6 \% \mathrm{GA}$ and $1 \mathrm{mg} / \mathrm{ml}$ ruthenium red. Following that, they were rinsed rapidly in $0.01 \% \mathrm{Knop}$ solution then postfixed for $30 \mathrm{~min}$ with $1 \% \mathrm{OsO}_{4}$ fixative.

In the assay of calcium cytochemistry, postfixation was made with a mixture of $\mathrm{OsO}_{4}$ and potassium pyroantimonate at the respective final concentrations of $1 \%$ and $50 \mathrm{mM}$. The fixed samples were washed in fresh inorganic culture medium then dehydrated through a graded ethanol series for 15 min each, after which they were embedded in Spurr's low viscosity resin (10). Ultrathin sections were cut on a Porter-Blum ultramicrotome (MT-1) then stained with $3 \%$ uranyl acetate in $50 \%$ ethanol for 6 min followed by a lead citrate strain (4) for $3 \mathrm{~min}$. Observations were made with a transmission electron microscope (JEOL, JEM-100S).

\section{RESULTS}

A cross section through the proximal part of an axopodium of Echinosphaerium akamae is shown in Fig. 1. A bundle of contractile tubules (arrow) with outer diameters of 10 to $25 \mathrm{~nm}$ each ( $21 \mathrm{~nm}$ on the average, $\mathrm{n}=27$ ) can be seen near the axonemal microtubules. These tubules run parallel to the longitudinal axis of the axopodium, and therefore, to the axonemal microtubules as reported by Shigenaka et al. (9) and Suzaki et al. (11). When the heliozoans were stimulated by giving them food organisms, their contractile tubules took on peculiar shapes, formerly called granular X-bodies. The axonemal microtubules folded or disintegrated at the same time.

We believe that the intermediate stage in this contraction process is shown in Fig. 2 because both granular (large arrow heads) and twisted tubular forms are present.

Fig. 1. Cross section through a proximal portion of a normal axopodium. Ax, axoneme; M, mitochondrion; arrow, contractile tubules. Inset: high magnification of the cross-section of the contractile tubules. $\times 37,000$; inset, 80,000 .

Fig. 2. The appearance of contractile tubules during axopodial contraction. Heliozoans were stimulated with the food organism Chilomonas paramecium. Ax: axoneme; large arrows: slightlytwisted contractile tubules; small arrow: just-twisting wavy configuration of the contractile tubules. $\times 80,000$. 


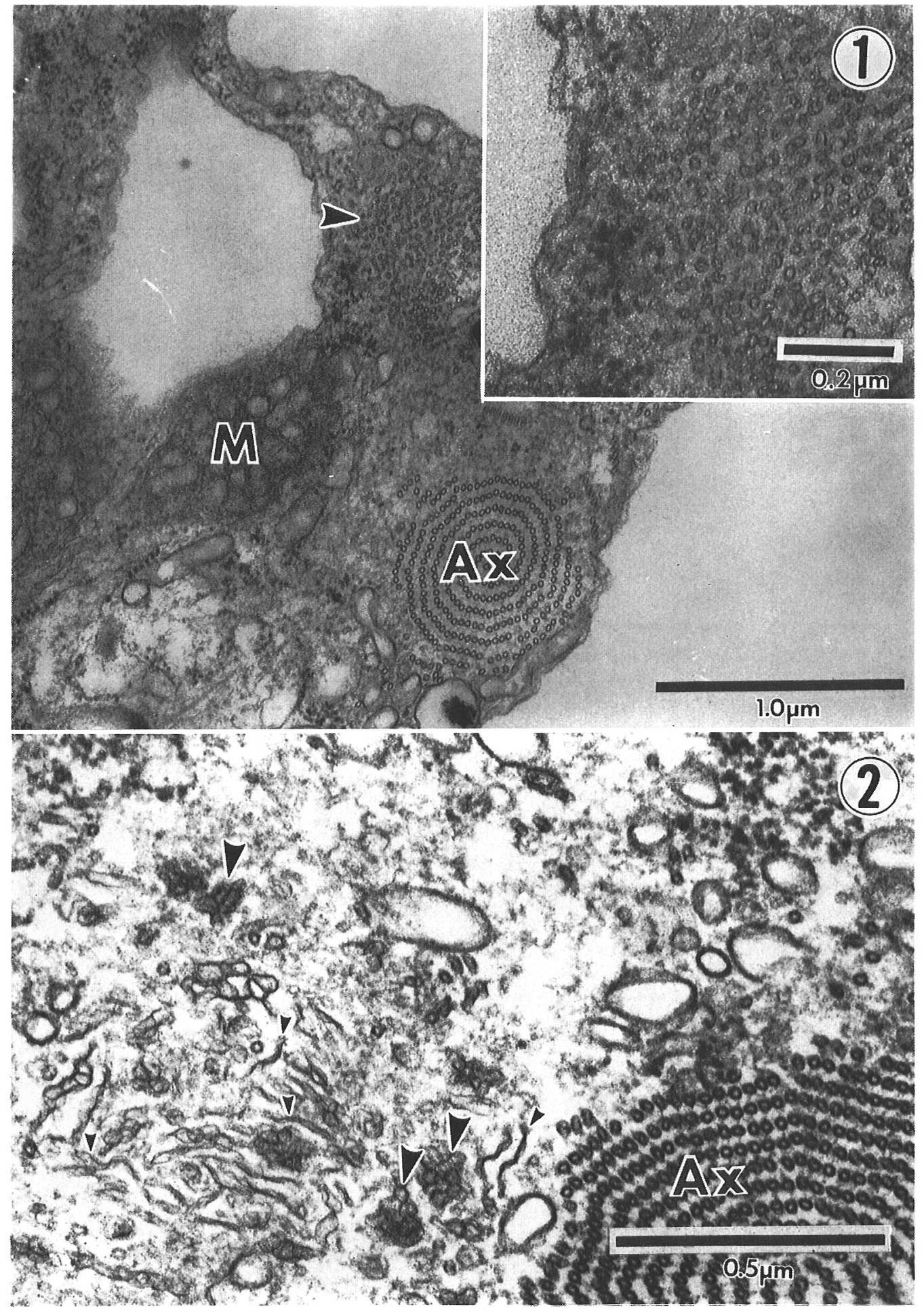




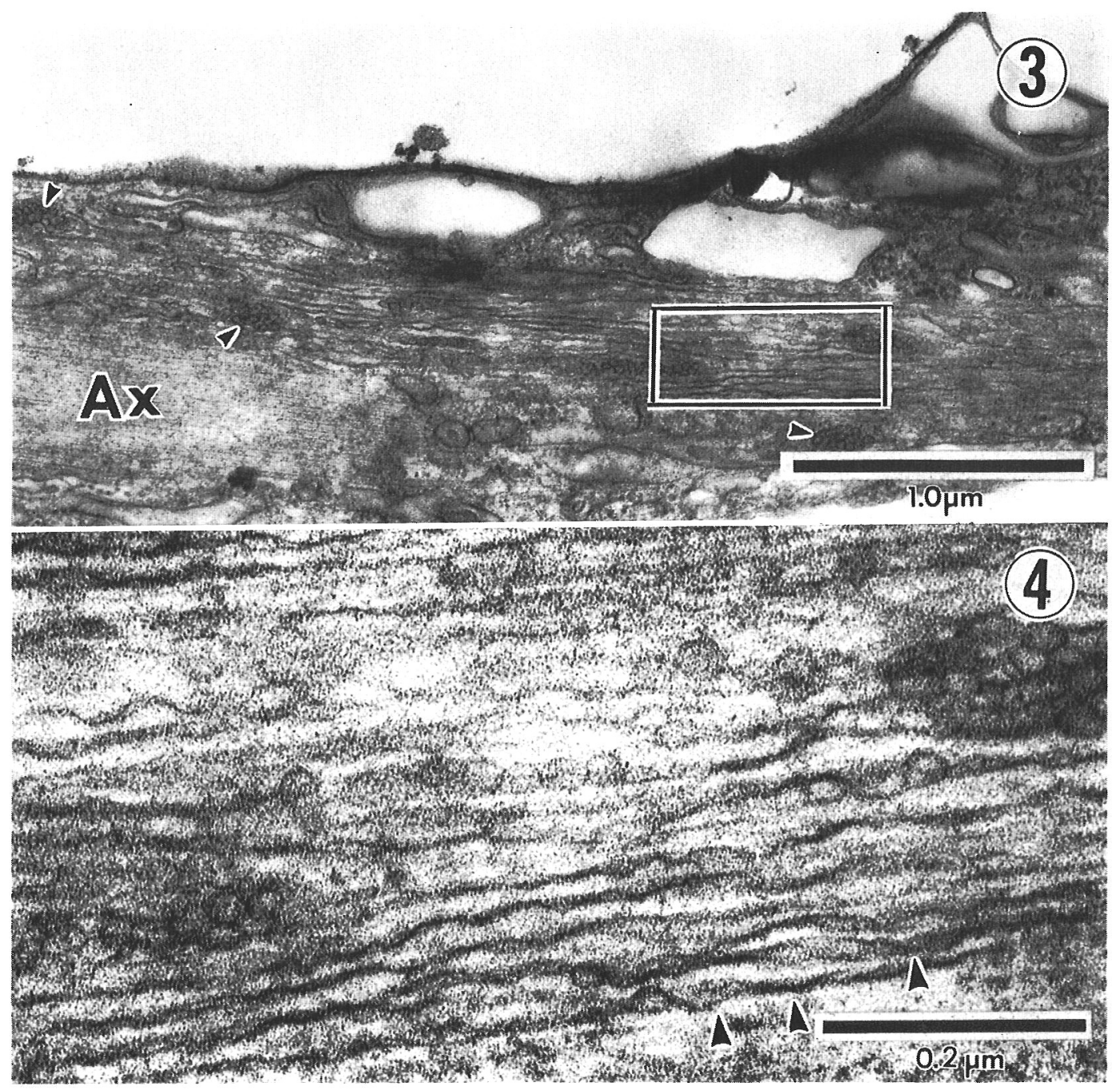

Fig. 3. Longitudinal section of an axopodium that contains just-twisting and/or coiling contractile tubules. Ax: axoneme; arrows: already twisted contractile tubules. $\times 37,000$.

Fig. 4. High magnification of the axopodial region shown in Fig. 3 (frame). Arrows: justtwisting contractile tubules. $\times 163,000$.

Figs. 5, 6. Longitudinal (Fig. 5) or cross-sections (Fig. 6) of axopodia showing the coagulated stage of the contractile tubules. Ax: axoneme. $\times 17,000$ and 37,000.

Figs. 7-9. Longitudinal (Fig. 7) or cross-sections (Figs. 8, 9) of axopodia in stimulated heliozoans that were postfixed in the presence of potassium pyroantimonate $(50 \mathrm{mM})$ and $\mathrm{OsO}_{4}(1 \%)$. Dense deposits resulted from Ca-Sb precipitation. Figs. 7 and 8 apparently show the early stage of the twisting of contractile tubules (see the text). Ax: axoneme; M: mitochondria. $\times 34,000$. 


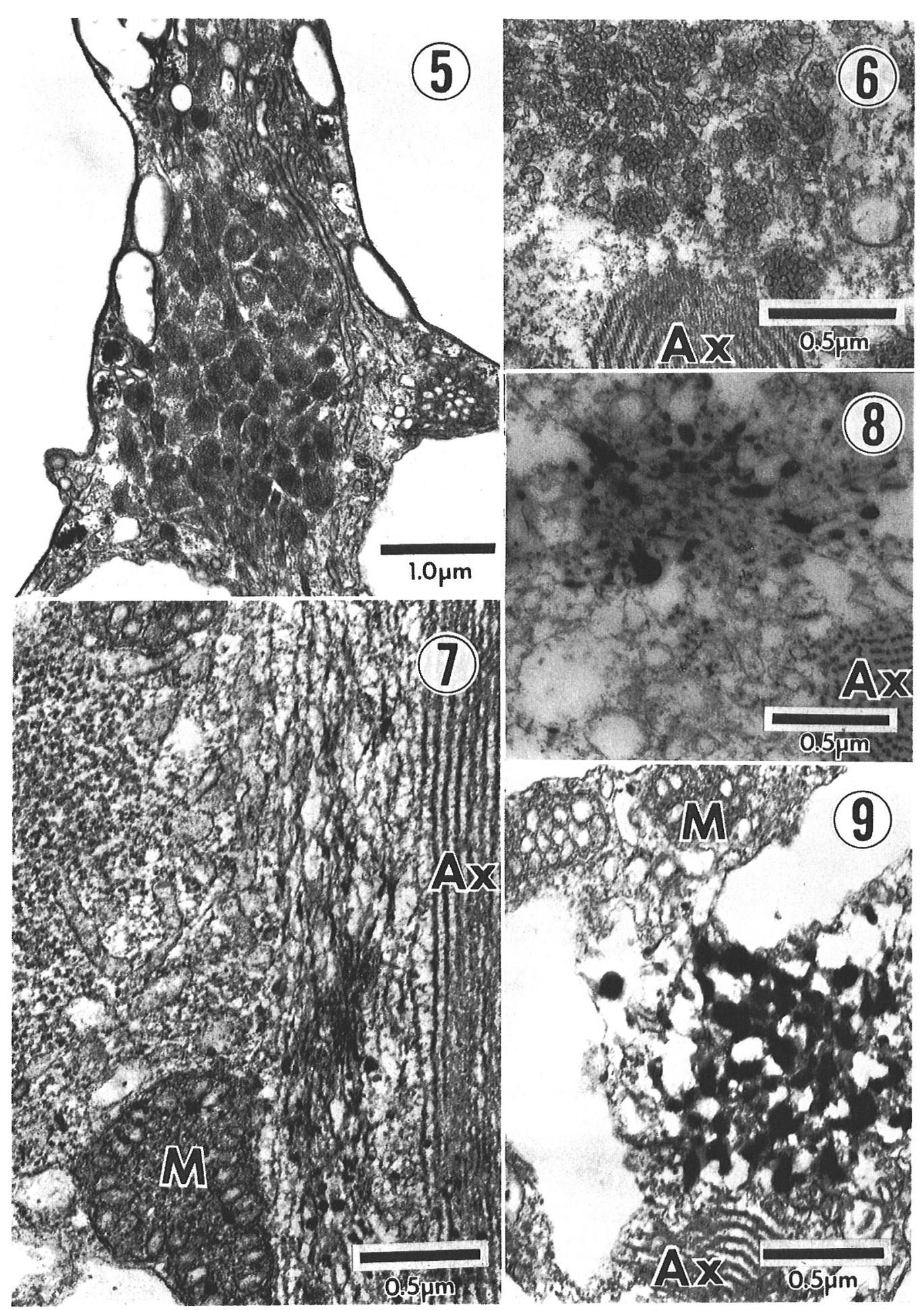


Also, filamentous structures (small arrow heads) that may be derived from the tubular forms due to twisting and/or coiling are present. Longitudinal sections of the contractile tubules show that they are just changing to granular structures (Figs. 3 and 4). The tubules appear to twist (Fig. 4, arrows), coil and, finally, interwind. The granular form (Fig. 3, arrows) of the contractile tubule may be produced by the interweaving of several twisting tubules. Longitudinal and cross sections of completely coagulated contractile tubules (the former granular X-bodies) are shown, respectively, in Figs. 5 and 6 . Note that difference can be seen between the cross and longitudinal sections of the coagulations, and that they are distributed only in the proximal regions of the axopodium.

A calcium cytochemistry assay, using the potassium pyroantimonate method, was made to examine the relation between alterations in $\mathrm{Ca}^{2+}$ localization and contractile tubules during axopodial contraction. No deposits were present on the contractile tubules in any heliozoan cell that had not been stimulated by food organisms. But, in stimulated cells a number of deposits appeared on the contractile tubules (Figs. 7-9). An early stage of axopodial contraction, in which deposits can be seen along the filamentous structures and on the axonemal microtubules is shown in Fig. 7. As the self-twisting and coiling of the contractile tubules proceeds, conspicuous large deposits accumulate on the tubules. The deposits at this stage (Fig. 8) appear on the filamentous or granular structures as in the contractile tubules shown in Fig. 2.

When the contractile tubules are coagulated completely, much larger deposits can

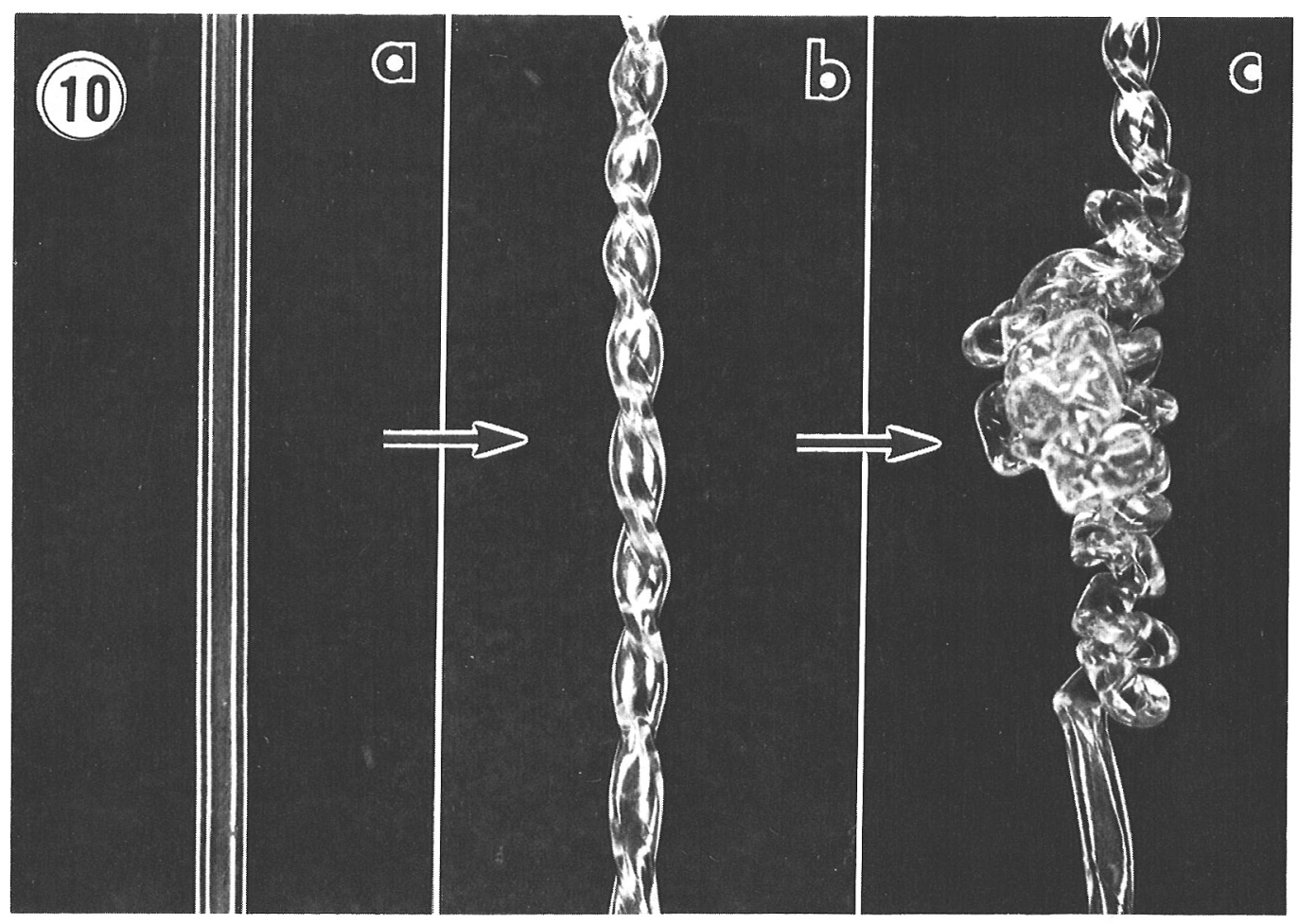

Fig. 10. A model of the contraction of contractile tubules. Every tubule is believed to shorten by twisting and/or coiling in response to an increment of $\mathrm{Ca}^{2+}$ ions. 
be seen on all the coagulated units that do not contain filamentous deposits (Fig. 9). The deposits shown in Fig. 9 seem to correspond to the proposed granulated form (9) (Fig. 6). Based on our detailed electron-microscopy, we propose a model with which to understand the contraction mechanism of contractile tubules. It is shown in Fig. 10. Contraction is considered to be caused by the twisting and/or coiling of the tubules, which may be drived by $\mathrm{Ca}^{2+}$-dependent conformational changes in the subunits that make up the contractile tubules.

\section{DISCUSSION}

Contractile tubules, previously called X-bodies (8) have been suggested to be contractile elements that cause axopodial contraction or flow $(9,11)$. But, the intermediate stage in this structural change does not show up when the contractile tubules shorten. The study reported here demonstrates that these structures apparently are transformed to the so-called granular form by their own twisting and/or coiling during the axopodial contraction or flow that takes place when food organisms first become attached to the axopodial tip. In this way, the twisting or coiling of every tubule is believed to enforce the shortening of the axopods, therefore, we propose "contractile tubule" as the correct name for this organelle.

In some peritrichous ciliates (Vorticella, Carchesium, Zoothamnium), the spasmoneme that functions in stalk contraction contains filaments that are 2 to $3 \mathrm{~nm}$ each in diameter (1). But, in the heterotrichous ciliate, Stentor, the extended myoneme consists of longitudinally oriented myofilaments approximately $4 \mathrm{~nm}$ in diameter (2); and, the extended, contractile flagellar root is composed of filaments $(5-8 \mathrm{~nm}$ in dia) that run parallel to the axis of the structure (5-7). In the study reported here the contractile elements were characterized by a bundle of tubular forms with outer diameters of about $21 \mathrm{~nm}$; these were oreinted longitudinally along the axonemal microtubules just beneath the axopodial membrane. The tubular appearance and the diameter differ from those of the contractile filaments of other contractile protozoans. These contractile tubules also differ from actin filaments morphologically. Therefore, we believe this to be the first time that this kind of contractile element has been described in any eukaryote.

Calcium cytochemistry indicates that intracellular $\mathrm{Ca}^{2+}$ concentrations increase and that calcium is supplied to the contractile tubules once the axopodia are stimulated to contract. Our results supply evidence that the twisitng and/or coiling of these tubules may be mediated by substructural or conformational changes in the tubules themselves. A similar contraction mechanism has been suggested in filaments consisting of the contractile flagellar roots of the flagellate, Tetraselmis (6); but, the configurations of the two contractile elements differ. Structural changes such as twisting and coiling apparently generate enough of a motive force to shorten individual filaments. We also found a large amount of calcium inside the vesicles that surround the axonemal microtubules (in preparation). Consequently, $\mathrm{Ca}^{2+}$ probably are supplied to the contractile tubules as well as to the axonemal microtubules when the ion is released from calcium-sequestering vesicles.

Another possibility also must be considered, that there may be an influx of $\mathrm{Ca}^{2+}$ ions across the axopodial membrane from the external medium that would cause twisting of the contractile tubules and/or disassembly of the axonemal microtubules because there is hardly any axopodial contraction in $\mathrm{Ca}^{2+}$-free medium $\left(10^{-7} \mathrm{M}\right.$ or 
less) or in the presence of $\mathrm{Mn}^{2+}\left(10^{-3} \mathrm{M}\right)$, a presumed inhibitor of $\mathrm{Ca}^{2+}$ channels (9).

\section{REFERENCES}

1. Amos, W.B. Contraction and calcium binding in the vorticellid ciliates. in Molecules and Cell Movement, ed. S. Inoué and R.E. Stephens, Raven Press, New York, pp. 411-436, 1975

2. HuANG, B. and D. MaZia. Microtubules and filaments in ciliate contractility. in Molecules and Cell Movement, ed. S. Inoué and R.E. Stephens, Raven Press, New York, pp. 389-409, 1975

3. OCKLEFORD, C.D. and J.B. TUCKeR. Growth, breakdown, repair, and rapid contraction of microtubular axopodia in the heliozoan. Actinophrys sol. J. Ultrast. Res. 44, 369-387, 1973

4. Reynolds, E.S. The use of lead citrate at high $\mathrm{pH}$ as electron-opaque stain in electron microscopy. J. Cell Biol. 17, 208-212, 1963

5. Salisbury, J.L. and G.L. Floyd. Calcium-induced contraction of the rhizoplast of a quadriflagellate gree alga. Science 202, 975-977, 1978

6. Salisbury, J.L. Calcium-sequestering vesicles and contractile flagellar roots. J. Cell Sci. 58, $433-443,1982$

7. Salisbury, J.L. Contractile flagellar roots; the role of calcium. J. Submicrosc. Cytol. 15, 105110,1983

8. Shigenaka, Y. and M. Kaneda. Studies on the cell fusion of heliozoans. IV. An electron microscopical study on the fusion process accompanied with axopodial degradation. Annot. Zool. Japon. 52, 28-39, 1979

9. Shigenaka, Y., K. Yano, R. Yogosawa and T. Suzaki. Rapid contraction of the microtubulecontaining axopodia in a large heliozoan Echinosphaerium. in Biological Functions of Microtubules and Related Structures, ed. H. Sakai, H. Mohri and G.G. Borisy, Academic Press, Tokyo, pp. 105-114, 1982

10. SpURR, A.R. A low-viscosity epoxy resin embedding medium for electron microscopy. $J$. Ultrast. Res. 26, 31-43, 1969

11. Suzaki, T., Y. Shigenaka, S. Watanabe and A. Toyohara. Food capture and ingestion in the large heliozoan. Echinosphaerium nucleofilum. J. Cell Sci. 42, 61-79, 1980

(Received for publication, February 2, 1985) 